\title{
OBJECIÓN DE CONCIENCIA, LA MUERTE Y EL MORIR EN ENFERMEDADES EN ETAPA TERMINAL
}

\author{
Octaviano Humberto Domínguez Márquez*
}

\begin{abstract}
Resumen: Se realiza un análisis crítico de la figura de la objeción de conciencia como una expresión de la consecución de nuevos espacios de libertad en sociedades en continua evolución, las que sin embargo acusan rezagos, específicamente en el campo de la salud.

Por otra parte, se enlaza la objeción de conciencia con los aspectos del final de la vida y el proceso tanatológico, en las encrucijadas éticas que la tecnología moderna plantea al cambiar drásticamente la forma de morir.
\end{abstract}

Palabras clave: objeción de conciencia, tanatología

\section{CONSCIENTIOUS OBJECTION, DEATH AND DYING IN TERMINAL ILLNESSES}

\begin{abstract}
This paper carries on a critical analysis of the figure of conscientious objection as an expression of the achievement of new spaces of liberty in societies in continuous evolution, that however, are still somewhat behind the times, specifically in the area of health.

In addition, it links conscientious objection with aspects of the end of life and the tanatological process, in the ethical puzzles that modern technology presents by changing drastically the way we die.
\end{abstract}

Key words: conscientious objection, tanatology

\section{OBJEÇÃO DE CONSCIÊNCIA, A MORTE E O MORRER COM DOENÇAS EM FASE TERMINAL}

Resumo: Faz-se uma análise crítica da figura da objeção de conciencia como uma expressão da conquista de novos espaços de liberdade em sociedades em contínua evolução, específicamente no campo da saúde. Por outro lado, aproxima-se a objeção de conciência com os aspectos de final de vida e processos tanatológico, nas encruzilhadas éticas que a tecnologia moderna levanta ao mudar drásticamente a forma de morrer.

Palavras chave: objeção de conciência, tanatología

\footnotetext{
Médico Cirujano y Partero. Profesor de pre y posgrado en Bioética, Escuela Superior de Medicina del Instituto Politécnico Nacional, México Correspondencia: octavidm@yahoo.com.mx
} 


\section{Introducción}

Una gran falla del desarrollo de la humanidad es estar al margen del aprendizaje del morir. Es incalculable todo lo que se ha escrito, hablado y realizado en torno al evento muerte $y$, sin embargo, puede afirmarse que aún estamos lejos de estar conscientes de la aceptación de ese fenómeno universal y absoluto. Expresó Eduardo Césarman: "es más probable estar muerto que vivo"(1).

A esta no aceptación se agrega la negativa de diversas instancias médicas, particularmente en pacientes terminales, para aplicar un procedimiento eutanásico ante un mandato legal o una orden superior, porque ello afectaría sus convicciones, creencias religiosas o ideologías. Esa resistencia, llamada "objeción de conciencia”, tiene innumerables implicaciones en los campos clínico, epidemiológico, jurídico o social, e inclusive repercusión política en la comunidad.

La objeción de conciencia implica un reconocimiento del valor de la individualidad más que de las minorías, ya que el Estado que la legitima y legaliza imprime con ello un sentido democratizante a su régimen.

Los reclamos sociales en lo político han evolucionado desde hacerse oír en masa, avanzar luego en la consecución del reconocimiento legal en los derechos de las minorías o de grupos tradicionalmente marginados, hasta reconocer el alto significado de las determinaciones individuales de la conciencia que influyen en la dinámica social y política.

En el campo de la salud son múltiples y variados los casos en los cuales las complicaciones tecnológicas propician el encuentro de la objeción de conciencia. Algunos de ellos son el aborto, la eutanasia, los procedimientos para desconectar a un enfermo declarado con muerte cerebral, entre otros. Así también en la oposición para atender un número exagerado de pacientes y en la resistencia a ser el brazo ejecutor de los pacientes terminales, a quienes les ha sido negada la mínima asistencia tanatológica y los cuidados paliativos humanizantes, como sucede además con la obstinación terapéutica en procedimientos fútiles. También en el área farmacéutica, en cuanto a la producción, distribución y suministro de medicamentos con potencialidad abortiva. Además de los casos muy conocidos de los Testigos de Jehová.
Desde luego, existen personas que racionalmente esperan la muerte, otras fuera de la racionalidad la buscan y las menos que entregadas a su fe construyen gustosamente el puente psicorracional-espiritual para aceptar el final de su vida.

El morir ha cambiado(2). La tecnología y las instituciones de atención médica revolucionan la concepción de la muerte y el morir. La humanidad, a través de la ciencia médica, desarrolla procedimientos inverosímiles para mantener con vida a las personas, llegando al extremo de ejercer el ensañamiento terapéutico con tal de sostener lo más posible la ilusión de la familia de que su paciente aún vive. Sin embargo, habrá otro que objete y disienta por motivos de conciencia, para no prolongar una agonía con elementos fútiles. $\mathrm{O}$, en su caso, para no aplicar procedimientos eutanásicos.

\section{La objeción de conciencia, su desarrollo y la tana- tología}

Es interesante anotar que la objeción de conciencia es reconocida desde hace muchos siglos, enfocada principalmente a la defensa de creencias religiosas, consideradas como un valor fundamental de grupo y de carácter individual. También como ejemplo de defensa heroica, anteponiendo el honor y la religiosidad para disentir ante imperativos abrumadores. Tal es el caso de Antígona, en su enfrentamiento desigual con el tirano. En defensa de sus creencias religiosas, Antígona desobedece sus órdenes para dejar insepulto a su hermano, en conocimiento de que en ello le iba la vida.

En el siglo pasado, hay evidencia de objeción de conciencia con relación al desempeńo militar, primero respecto de la participación directa en las guerras y, a mitades del siglo, en la incorporación de conscriptos, con una extensión a las actividades paramilitares o en relación con la acción militar.

El traslado de la objeción de conciencia de las áreas religiosas a otro campo se da alrededor de 1847 con la invasión del ejército de Estados Unidos a México, en una protesta unipersonal(3) de Henry David Thoreau, negándose a pagar impuestos como recriminación de que iban a ser utilizados en esa guerra injusta.

Es en Europa, principalmente en España e Italia, donde la resistencia a la conscripción de los jóvenes es muy fuerte, desafiando en el primer caso a la dictadura franquista, a tal grado que no importó a ésta una importante recomendación de la Asamblea Constitutiva 
del Consejo de Europa(4) para reconocer el derecho a la objeción de conciencia y dejar en libertad a los jóvenes apresados purgando condena.

Después aparece en el campo de la salud con relación al aborto y progresivamente en un sinnúmero de procedimientos que fueron considerados lesivos para convicciones o creencias religiosas. Además, se extiende a la resistencia por motivos de lesión a valores profesionales o, simplemente, a la defensa de otros valores, como en el caso de la eutanasia, la desconexión de un respirador y el exceso de consulta, en los que se defiende la vida y el bienestar de los pacientes.

Otra extensión de la objeción de conciencia se refiere a los farmacéuticos en España, que expresan su negativa a la producción, distribución y suministro de ciertos medicamentos, en particular, la píldora del día después y otros de carácter abortivo.

Resulta pertinente señalar las diferencias entre la objeción de conciencia y la desobediencia civil. La primera es generalmente de manifestación individual, por parte del personal de salud, de algún paciente o de sus familiares; la segunda tiene una dimensión social más amplia y se caracteriza por ser una movilización de grupos, no violenta, como rechazo a una ley que se considera lesiva a intereses económicos o sociales, u ofensiva a ciertos hábitos y costumbres. Implica la negativa a obedecer los términos procedimentales, obstruyendo en lo posible su aplicación(3).

No es frecuente que el proceso de morir en los casos terminales sea conducido con un método tanatológico humanista. La confusión de lo auténticamente humanista está en que en la mayoría de los casos predomina la compasión y la pronta atención a las necesidades del enfermo y eso no basta para imprimir el verdadero valor de una atención tanatológica merecida para un ser humano.

Es necesario aprender a morir e ir más allá de los pasos del proceso que invoca Kubbler Ross(5). Llegar a la etapa final de aceptación en la que debe darse la construcción de un puente psicológico, racional y espiritual, no sólo de mansa resignación sino de un esclarecimiento consciente del significado de la muerte en uno mismo y en la humanidad toda. La educación en la nińez, en lugar de ocultar lo relativo a la muerte real, debiera informar de la racionalidad del fenómeno y cómo prepararse para enfrentarlo en caso necesario.
A pesar de las turbulencias psicológicas desatadas ante el diagnóstico de enfermedad terminal, es posible conseguir un aprendizaje del morir. Como proceso complejo que oscila entre la razón y los temores espirituales, conseguir el equilibrio entre una razón suficiente y una plenitud espiritual lleva a la tranquilidad y la paz interior del fondo de la psique, con el fin de aceptar mejor la intensidad del dolor y la enajenación del sufrimiento.

Aprender a morir aleja con firmeza las tentaciones eutanásicas y de suicidio asistido, muy frecuentes en los enfermos terminales. Nadie merece ser muerto por otro semejante, nadie debe pedir a otro que lo mate y nadie debe atribuirse la facultad de hacerlo. Bien dice Diego Gracia(6): "La naturaleza, hoy como siempre, es la que acabará haciendo lo demás. La naturaleza, no el médico".

De acuerdo con los esquemas actuales, pese a la vigencia de la ley de la eutanasia, también el personal de salud tiene el derecho de evadir la ejecución por respeto a sus convicciones ideológicas, profesionales o religiosas. La objeción de conciencia se extiende a los familiares, quienes también tienen derecho a oponerse a los actos eutanásicos y a los tratamientos colmados de futilidad que su conciencia rechaza.

\section{La tanatología de hoy}

La asistencia tanatológica siempre estuvo sostenida por las creencias religiosas, que actuaron para bien de los creyentes y para complicar con importantes sentimientos de culpa a los no creyentes.

En la mayor parte de los países de Iberoamérica, la religión dominante se ha mezclado con otras creencias de los diferentes grupos indígenas, es decir, ha habido dos concepciones distintas del proceso de morir y del culto a los muertos. Sin embargo, se reconoce que estos grupos aceptan de mejor manera la presencia de la muerte, con mayor entereza y resignación. La enfermedad y la muerte son tomadas con fatalidad, sin entrar en procedimientos que prolongan la agonía o dolerse con exageración por la pérdida de sus familiares.

La muerte de un miembro de la familia en nuestra cultura es tomada de manera diferente si se trata de un recién nacido, infante, adolescente, adulto joven o anciano. Al recién nacido y al infante los consideran como angelitos, inocentes de toda culpa, y a los 
ancianos como individuos que ya vivieron lo que les correspondía, en cuyo caso la resignación de los familiares es pronta. En cambio, un adulto en la plenitud de la vida es una pérdida muy sentida, por los muchos intereses que se pierden y los afectos truncados que se encuentran en su apogeo.

La presencia de cáncer o SIDA en un niño es considerada como injusta y con una fuerte repercusión afectiva, por lo tanto, la compasión abarca prácticamente todos los espacios de la atención tanatológica. Para estos casos, la psicoterapia no sólo se dirige a los nińos afectados orgánicamente por el padecimiento terminal, sino al personal de salud que sabe de la muerte próxima de los enfermos y no sabe con precisión cuál debe ser su papel frente a ellos. La tanatología de hoy involucra las intervenciones psicoterapéuticas con el reconocimiento de indispensables. El rescate de la subjetividad de las personas para llevarlas a un equilibrio estable y armónico debe ser cuidadosamente planeado, integrando al enfermo, sus familiares y el personal de salud no importando la edad de la persona.

Actualmente, la tanatología considera un cúmulo sistematizado de cuidados paliativos con el fin de sostener y proporcionar la mejor calidad de vida posible, atendiendo al binomio paciente-asistente o cuidador, ya sea en fases iniciales o en fase terminal. Estos procedimientos pretenden acercar al paciente y sus familiares los mayores beneficios de acuerdo con sus necesidades más significativas.

La psicoterapia, la razón y la espiritualidad son aportes diferentes. Esta tríada es la columna vertebral de la tanatología moderna, excepto que la última puede estar manifiesta o no en algunos individuos o simplemente no ser aceptada. El manejo puede ser simultáneo y, de hecho, conseguir el dominio de la psique facilita la aceptación de los razonamientos y viceversa. La construcción de un puente armónico hacia el deceso radica en comprender la finitud humana y darle un sentido a la muerte, idóneamente y desde un comportamiento cotidiano.

El punto cardinal, de mayor significado en todo el proceso es la transformación de ese potencial de energía que se expresa en el temor y los miedos a dejar de ser, a pasar a un estadio desconocido, a expiar las culpas sin preparación, a ser el juez de su propia conciencia, a dejar de estar en un escenario en el que se considera necesario o indispensable. Esto genera un estado alar- mante de angustia, con alternancias depresivas y un deseo imperativo de encontrar la paz de la psique, de la conciencia y del espíritu.

Erich Fromm(7) concibe la libertad como problema psicológico; a pesar de ese andar incesante del hombre en su conquista, cuando finalmente la encuentra se queda inmóvil, le atemoriza ejercerla, prefiere continuar en una cómoda sumisión. A través de su historia, el hombre ha sabido que puede luchar contra la naturaleza y dominarla; oponerse a la Iglesia y ganar su derecho a la libertad de creencias y enfrentarse al Estado absolutista y democratizarlo. Sin embargo, aunque aún no conquista todos los espacios de libertad a los que tiene derecho, tiene la seguridad de que nunca le ganará a la muerte. Podrá aumentar los plazos, pero no conseguirá ser inmortal o, por lo menos, como muchos desean, vivir una larga vida. No le consuela que sea igual para todos los seres vivos; es su caso y, por tanto, su miedo, su angustia y sus temores.

La tanatología aprecia los conflictos modernos del hombre, sus avances en la ciencia y la tecnología y, desde luego, la concepción diversa sobre la muerte. Si la esperanza de vida es de 78 años para la mujer y 74 para el varón, quiere decir que se han librado de una gran cantidad de padecimientos por los que morían poblaciones por millones $\mathrm{y}$, por lo tanto, el espectro de la muerte no es el mismo que en los inicios del siglo $\mathrm{XX}$, con una mortalidad en promedio alrededor de los treinta ańos de edad.

A pesar del crecimiento de los servicios de salud y de una atención en el final de la vida otorgada en los hospitales, la tanatología no ha sido incorporada como parte ineludible del tratamiento clínico. Lo que se hace actualmente es motu proprio del personal de salud, especialmente del médico y de la enfermera, de una manera empírica, con buena intención de apoyar al paciente y a sus familiares, pero sin ningún método. Por otra parte, no existe la especialidad como tal entre el personal de salud.

Hay avances importantes en cuanto a la reconceptualización de la tanatología. Está pasando de una fase empírica -de carácter compasivo- a la profesionalización y formalidad de su integración en los planes de estudio del médico, la enfermera, el psicólogo y el trabajador social, con un enfoque multidisciplinario, situación que modifica radicalmente la posición solitaria del médico y la fortalece. Además, integra los aspectos psicológicos 
a la racionalidad del fenómeno e incorpora el enriquecimiento de la espiritualidad.

\section{La muerte y el morir. El trance tanatognomónico}

Los últimos momentos de vida suelen ser muy significativos. Las actitudes del paciente pueden ser de angustia y desesperación o de plenitud y paz. Se habla en los medios hospitalarios de un momento de lucidez anterior al deceso; la muerte se siente y con ella un final aceptado o, en otros casos, cuando la agonía es duradera, a algunos pacientes les cuesta morir.

Se conoce al momento próximo a morir como el "trance tanatognomónico", es decir, característico de la cercanía de la muerte. En las defunciones con sufrimiento y dolores intensos la persona es sometida a potentes analgésicos y sedantes, por lo que este momento se pierde. En caso de que el paciente esté consciente, sus sufrimientos se recrudecen y suman ante la clara percepción de ese momento final.

Los enfermos solicitan la presencia de la familia, piden algunas cosas en especial, se despiden, distribuyen algunos bienes, ajustan asuntos pendientes, piden ayuda espiritual, ya como continuación o de inicio, y quedan en espera de la muerte que suele ocurrir en un corto lapso. Para algunos, esos momentos previos al desenlace exponen, en toda su verdad, la esencia de la persona: ya no hay nada que ocultar ni que perder, se actúa sin la última máscara.

Los sentimientos que se desbordan en el momento de morir, son inocultables. Castilla del Pino(8) nos habla de su fundamento y de su dificultad para inhibirlos, tanto el odio y el amor como la simpatía y el rechazo. En el paciente terminal hay variadas manifestaciones. Los sentimientos íntimos, que algunos guardan de una manera acorazada, y lo que otros exponen abiertamente. Las condiciones de estrés y de depresión son síntomas alternativos en estrecha vinculación con el impacto de los sentimientos fuera de control. En ese agitado mar de sentimientos, jes posible conseguir una regulación aceptable?

El cultivo de la racionalidad en el paciente terminal no se contrapone con el desarrollo de la espiritualidad, de acuerdo con la religión que profese el enfermo. Para los creyentes, la espiritualidad significa un soporte que pueden elevar ante lo inexplicable. La racionalidad ante la muerte y el morir debe aplicarse con todo el cuidado necesario para no confrontarla con la espiritualidad. Lo que menos se espera es producir más agitaciones propiciadoras de confusión. La tanatología debe facilitar la expansión espiritual del enfermo, quitando trabas que impidan la negación, fenómeno frecuente en el complejo proceso del aprendizaje para la muerte y el morir.

\section{La objeción de conciencia y el morir}

Ante la presencia de un paciente diagnosticado con muerte cerebral y un cuerpo vivo (tanto que algunos de sus órganos servirán para renovar la vida de un paciente grave), algunos miembros del personal de salud y el propio médico se niegan a desconectarlo aduciendo sus propias convicciones.

La objeción de conciencia es un derecho plenamente establecido en muchos países desde hace algún tiempo, principalmente ligado a la relación laboral, como en el caso del aborto(9), situación estudiada por la Organización Internacional del Trabajo, desde hace ya varios años. Por otra parte, la "Encyclopedia of Bioethics" (10) menciona la dualidad que puede existir en esa resistencia objetora para participar, por ejemplo, en procedimientos de inseminación artificial, abortivos, eutanásicos o de encarnizamiento terapéutico en los pacientes, y luego negarse a procedimientos diagnósticos o de tratamiento, invocando principalmente creencias religiosas.

Esta objeción es reconocida como un derecho en cuanto su ejercicio no afecte o lesione a terceros. En el caso que nos ocupa, respecto de pacientes con muerte cerebral, la negativa a desconectarlos puede ser de parte del personal de salud como de los familiares y, en ese caso, la situación de perjuicio se registra en la prolongación de la estancia hospitalaria.

Finalmente, en ese proceso de la muerte y el morir se conjugan valores y derechos que la bioética desglosa y atiende: desde la autonomía que respalda la capacidad objetora por motivos de conciencia, la dignidad ineludible para decidir ese momento único e irreversible del morir, hasta la plena objeción de conciencia en esa marańa de complicaciones dadas por la utilización anárquica de la tecnología en el rezago de las normas y leyes específicas. 


\section{Referencias}

1. Césarman E. Hombre y Entropía. México: Pax México; 1974.

2. Llano E. El morir humano ha cambiado. Boletín Ofcina Sanitaria Panamericana 1990; 108(5-6): 465.

3. Barry P, Linzey A. Civil Desobedience. Diccionary of Ethics, Theology and Sociology. London/New York: Routledge; 1996: 152-153.

4. Asamblea Consultiva del Consejo de Europa. Convención Europea de los Derechos del Hombre. Madrid; 1967: 74.

5. Kübler Ross E. Sobre la muerte y los moribundos. Barcelona: Grijalbo/Mondadori; 2001.

6. Gracia D. Ética de los confines de la vida. Estudios de Bioética. Bogotá: El Búho; 1998: 312.

7. Fromm E. El miedo a la libertad. Buenos Aires: Paidós; 1971.

8. Castilla del Pino C. Teoría de los sentimientos. Barcelona: Tusquets; 2000: 63-64.

9. Organización Internacional del Trabajo. Problemas éticos. Problemas y situación de la legislación y de la práctica. En: Empleo y condiciones de trabajo en los Servicios Médicos y de Salud. Ginebra: OIT; 1985: 143-146.

10. May Th. Conscience. En: Post SG. Encyclopedia of Bioethics. $3^{\mathrm{a}}$ ed. Vol. I. USA: Macmillan Reference; 2004: 513516.

Recibido: 7 de julio de 2008

Aceptado: 15 de octubre de 2008 